\title{
Modeling and characterization of dielectrophoretically structured piezoelectric composites using piezoceramic particle inclusions with high aspect ratios
}

\author{
D. A. van den Ende, ${ }^{1,2,3, a)}$ R. A. Maier, ${ }^{4}$ P. L. M. J. van Neer, ${ }^{5}$ S. van der Zwaag, ${ }^{3}$ \\ C. A. Randall, ${ }^{4}$ and W. A. Groen ${ }^{1,3}$ \\ ${ }^{1}$ Holst Centre-TNO, HTC31, 5605 KN Eindhoven, The Netherlands \\ ${ }^{2}$ TNO (Netherlands Organization for Applied Scientific Research), Materials for Integrated \\ Products Department, Rondom 1,5612 AP Eindhoven, The Netherlands \\ ${ }^{3}$ Novel Aerospace Materials Group, Faculty of Aerospace Engineering, Delft University of Technology, \\ Kluyverweg 1, 2629 HS Delft, The Netherlands \\ ${ }^{4}$ Materials Research Institute, The Pennsylvania State University, University Park, Pennsylvania 16801, USA \\ ${ }^{5}$ TNO, Department of Process and Instrumentation Development, Stieltjesweg 1, 2628 CK Delft, \\ The Netherlands
}

(Received 10 October 2012; accepted 21 December 2012; published online 15 January 2013)

\begin{abstract}
In this work, the piezoelectric properties at high electric fields of dielectrophoretically aligned PZT_polymer composites containing high aspect ratio particles (such as short fibers) are presented. Polarization and strain as a function of electric field are evaluated. The properties of the composites are compared to those of PZT-polymer composites with equiaxed particles, continuous PZT fiber-polymer composites, and bulk PZT ceramics. From high-field polarization and strain measurements, the effective field dependent permittivity and piezoelectric charge constant in the poling direction are determined for dielectrophoresis structured PZT-polymer composites, continuous PZT fiber-polymer composites, and bulk PZT ceramics. The changes in dielectric properties of the inclusions and the matrix at high fields influence the dielectric and piezoelectric properties of the composites. It is found that the permittivity and piezoelectric charge constants increase towards a maximum at an applied field of around $2.5-5 \mathrm{kV} / \mathrm{mm}$. The electric field at which the maximum occurs depends on the aspect ratio and degree of alignment of the inclusions. Experimental values of $d_{33}$ at low and high applied fields are compared to a model describing the composites as a continuous polymer matrix containing PZT particles of various aspect ratios arranged into chains. Thickness mode coupling factors were determined from measured impedance data using fitted equivalent circuit model simulations. The relatively high piezoelectric strain constants, voltage constants, and thickness coupling factors indicate that such aligned short fiber composites could be useful as flexible large area transducers. (C) 2013 American Institute of Physics. [http://dx.doi.org/10.1063/1.4775815]
\end{abstract}

\section{INTRODUCTION}

Piezoelectric materials are used in many applications as sensing, actuating, or energy harvesting elements. Piezoelectric ceramics possess excellent electromechanical coupling coefficients but suffer from high density, poor mechanical properties, and difficult processing. Piezoelectric ceramicpolymer composites are a class of materials where a piezoelectric ceramic phase is embedded in a polymer matrix. The shape and connectivity pattern of the PZT phase not only determines the aforementioned properties ${ }^{1}$ but also the ease of processing. For instance, 1-3 composites can achieve higher coupling coefficients and voltage sensitivity than monolithic ceramics, but are generally difficult to process. This processing obstacle results in relatively costly materials which are less suitable for large scale cost effective manufacturing. Alternatively, low-cost composites such as 0-3 PZT particle-polymer composites are flexible, easy to process,

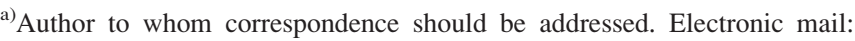
daan.vandenende@tno.nl.
}

and can be applied at low temperatures and in large areas. ${ }^{2}$ However, while the limited connectivity of the ceramic phase enables flexibility of the composites, it also causes these types of composites to suffer from inferior piezoelectric properties compared to their 1-3 counterparts. The decrease in properties is due to limited connectivity in combination with the mismatch in permittivity between the matrix and the piezoelectric inclusions. ${ }^{3,4}$

Dielectrophoresis (DEP) ${ }^{5}$ is a technique which allows in situ forming of quasi 1-3 structures from randomly oriented dielectric particles in a thermosetting resin. When an alternating electric field is applied during curing of the matrix, the dielectric particles in the composite coalesce to form chains, oriented in the direction of the applied field. ${ }^{6,7}$ Piezoelectric coupling in the direction of alignment increases for composites with alignment of equiaxed particles. ${ }^{8}$ Composites with aligned elongated particles, i.e., particles of aspect ratio higher than one, have also been assessed..$^{9-11}$ Piezoelectric composites with randomly oriented elongated particles inclusions demonstrate improved transducer properties. ${ }^{12}$ Low field piezoelectric constants of DEP aligned short fiber 
composites are significantly higher than conventional 0-3 composites. At low volume fractions of PZT, $d_{33}$ values can reach about 100 times the values of conventional 0-3 PZT composites with equiaxed particle inclusions. ${ }^{11}$ However, further insight is needed into the high field properties and resonance characteristics of such composites to determine their full potential as transducers. In this paper, a recently derived model for piezoelectric properties of DEP structured PZT-polymer composites ${ }^{11}$ is expanded for high field behavior and compared to experimental results. Additionally, results from resonance measurements are presented and compared to existing theory.

\section{THEORY}

The orientation of non-spherical, high-aspect ratio particles in a dielectric fluid will influence the electric field acting on them. Short fiber particles in a continuous matrix can be approximated by treating them as homogeneous ellipsoidal inclusions. The depolarization factor, $N$, for homogeneous ellipsoid with semi-axes $a, b, c$ in a uniform electric field is given by the following equation: ${ }^{13}$

$$
N_{i}=\frac{a b c}{2} \int_{0}^{\infty} \frac{d s}{\left(s+i^{2}\right) \beta} \quad(i=a, b, c),
$$

where $\beta^{2}=\left(s+a^{2}\right)\left(s+b^{2}\right)\left(s+c^{2}\right)$. For ellipsoids oriented with their long axis, $c$, parallel to the electric field direction, $i=c$.

\section{Aligned ellipsoidal particles in composites}

In the dilute limit, the relative electric field acting on an ellipsoidal inclusion with aspect ratio $A R=c / a \approx l_{2} / w_{2}$ (see Figure 1) oriented in the electric field direction, $\Gamma_{A R}$, is given by $^{14}$

$$
\Gamma_{A R}=\frac{E_{2, \text { dilute }}}{E_{\text {app }}}=\frac{\varepsilon_{1}}{\varepsilon_{1}+N_{z}\left(\varepsilon_{2}-\varepsilon_{1}\right)},
$$

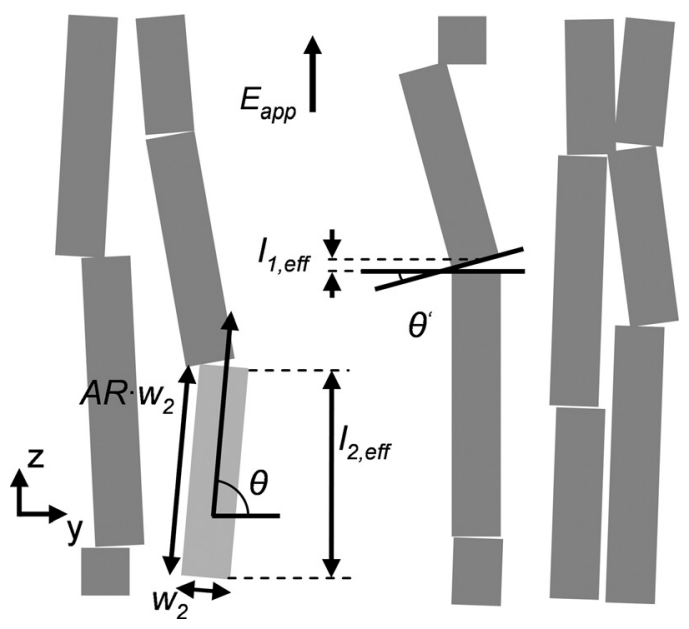

FIG. 1. Schematic of a cross section of the aligned fibers including the particle dimensions, $w_{2}, l_{2}\left(=A R \cdot w_{2}\right)$ and orientation angle, $\theta$, and the difference in angle between two fibers, $\theta^{\prime}$, from which the characteristic dimensions $l_{2, e f f}$ and $l_{1, \text { eff }}$ are derived. where $\varepsilon_{1}$ is the permittivity of the matrix and $\varepsilon_{2}$ is the permittivity of the ellipsoidal inclusion.

When the particles are in close proximity to each other, which is the case in non-dilute dielectrophoretically processed composites, interaction between the particles occurs. By treating the particle and matrix as two capacitors connected in series, the relative electric field acting on the particle, $\Gamma_{S}$, can be described using the size ratio between particle thickness, $l_{2}=A R \cdot w_{2}$, and thickness of the matrix layer in between the particles, $l_{1}$, which is defined as $R=\frac{l_{2}}{l_{1}}$ and the permittivity of the both phases

$$
\Gamma_{s}=\frac{E_{2, \text { series }}}{E_{\text {app }}}=\frac{(1+R) \varepsilon_{1}}{\varepsilon_{2}+R \varepsilon_{1}} .
$$

The combination of the effects described in Eqs. (2) and (3) gives the relation for the relative electric field acting on DEP structured ellipsoidal particles ${ }^{11}$

$$
\begin{aligned}
\Gamma_{e f f} & =\frac{E_{2}}{E_{a p p}}=\Gamma_{A R}+\left(1-\Gamma_{A R}\right) \Gamma_{s} \\
& =1-\frac{\left(N_{z, e f f}\left(\varepsilon_{1}-\varepsilon_{2}\right)^{2}\right)}{\left(\varepsilon_{2}+R_{e f f} \varepsilon_{1}\right)\left(\varepsilon_{1}+N_{z, e f f}\left(\varepsilon_{2}-\varepsilon_{1}\right)\right)} .
\end{aligned}
$$

Here, $N_{z, e f f}$ is the effective depolarization factor in the direction of the electric field and $R_{e f f}=\frac{l_{1, e f f}}{l_{2, e f f}}$ is the ratio of effective particle size over effective inter-particle distance. Both these parameters depend on the $A R$, of the particles and their average orientation, $\theta$ (see Ref. 11, for details).

Using the fact that the particle and matrix are connected in series, the (average) electric field acting on the matrix phase in the region between two particles, $E_{1}^{\prime}$, can be calculated

$$
\frac{E_{1}^{\prime}}{E_{a p p}}=\left(1-\Gamma_{e f f}\right) R_{e f f}+1 .
$$

Note that the electric field acting on the matrix region between the particles, $E_{1}^{\prime}$ is different than the electric field acting on the matrix region surrounding the particle chains, $E_{1}$, which is simply $E_{1}=E_{a p p}$ due to parallel connectivity.

Assuming a non-piezoelectric matrix, the equations for permittivity and piezoelectric charge constants of nonideally aligned high aspect ratio particle composites are ${ }^{11}$

$$
\begin{gathered}
\varepsilon_{D E P}=\varphi\left[\frac{\left(\Gamma_{e f f} \varepsilon_{2}-\varepsilon_{1}\right) \varepsilon_{2}}{\left(\varepsilon_{2}-\varepsilon_{1}\right)}-\left(\Gamma_{e f f} d_{332}\right)^{2} \frac{1-\varphi}{\varphi s_{1}+(1-\varphi) s_{332}^{\prime}}\right] \\
+(1-\varphi) \varepsilon_{1} \\
d_{33 D E P}=\left[\frac{\varphi s_{1}}{\varphi s_{1}+(1-\varphi) s_{332}^{\prime}}\right] \Gamma_{e f f} d_{332}
\end{gathered}
$$

where $\varphi$ is the PZT volume fraction, $s_{1}$ and $s_{332}$ are the compliances of the (isotropic) polymer and ceramic inclusions, $s_{332}^{\prime}$ is the equivalent compliance of the chains of particles, ${ }^{8}$ 
and $d_{332}$ is the piezoelectric charge constant of the ceramic inclusions.

The high field polarization, $P$, and strain, $S$, properties of piezoelectric 1-3 composites with continuous fibers ${ }^{15}$ as a function of applied field are

$$
\begin{aligned}
P\left(E_{\text {app }}\right)= & \varphi P_{2}\left(E_{\text {app }}\right)+[1-\varphi] P_{1}\left(E_{\text {app }}\right) \\
& -\left[\frac{\varphi[1-\varphi] d_{332}^{2}\left(E_{\text {app }}\right) E_{\text {app }}}{\varphi s_{1}+(1-\varphi) s_{332}^{E}}\right], \\
S\left(E_{\text {app }}\right)= & S_{2}\left(E_{\text {app }}\right)\left[\frac{\varphi s_{1}}{\varphi s_{1}+(1-\varphi) s_{332}^{E}}\right],
\end{aligned}
$$

where $P_{1}\left(E_{a p p}\right), P_{2}\left(E_{a p p}\right), S_{2}\left(E_{a p p}\right)$, and $d_{33_{2}}\left(E_{a p p}\right)$ are directly dependent on the applied field $E_{\text {app }}$. Equations (8) and (9) are obtained by multiplying the equations for $\varepsilon$ and $d_{33}$ of a 1-3 composite by the applied electric field. ${ }^{15,16}$ For DEP structured composites with high AR particle inclusions, the polarization and strain of the particle chains as a function of applied electric field can be calculated using the above equations by replacing $P_{2}\left(E_{a p p}\right)$ with $P_{2}^{\prime}\left(E_{a p p}\right), S_{2}\left(E_{a p p}\right)$ with $S_{2}^{\prime}\left(E_{\text {app }}\right)$, the effective polarization and strain of the aligned particle chains and $s_{332}$ with $s_{332}^{\prime}$ and $d_{332}$ with $d_{332}^{\prime}$, the effective compliance and charge constant of the DEP aligned particle chains. ${ }^{8}$

\section{Electromechanical coupling factors of DEP structured composites}

From impedance measurements, an estimate of the thickness mode coupling factor, $k_{t}$ can be made from the thickness resonance if the thickness of the sample is low compared to lateral dimensions. ${ }^{17}$ The $k_{t}$ can be calculated using the following equation: ${ }^{18}$

$$
k_{t}=\left(\frac{\pi}{2} \frac{f_{s}}{f_{p}} \tan \left[\frac{\pi f_{p}-f_{s}}{2} \frac{f_{p}}{f_{p}}\right]\right)^{1 / 2},
$$

where $f_{p}$ is the frequency at maximum resistance and $f_{s}$ is the frequency at maximum conductance.

\section{EXPERIMENTAL}

\section{Materials manufacturing}

PZT fibers were manufactured by wet spinning a PZT powder (PZT5A4, Morgan Electroceramics, Ruabon, UK) with a Cellulose Acetate binder (Acros Organics). The fibers were sintered at $1200{ }^{\circ} \mathrm{C}$ for $1 \mathrm{~h}$ in air in a closed $\mathrm{Al}_{2} \mathrm{O}_{3}$ crucible. PZT fibers were then broken to form high aspect ratio particles, with aspect ratios ranging from 5 to 15 . PZT fibers were mixed with a polyurethane (PU, Crystal Clear 202, Smooth-on Inc, Easton, USA) and dielectrophoretically processed during the curing stage of the polymer to form structured composites. The applied structuring electric fields ranged from $0.5 \mathrm{kV} / \mathrm{mm}$ to $4 \mathrm{kV} / \mathrm{mm}$ to achieve different levels of alignment. Further details of the procedure are described elsewhere. ${ }^{11}$ As reference samples, 1-3 composites with continuous fibers were manufactured using the same polyurethane matrix material in addition to bulk PZT ceramics. The bulk ceramic samples and fibers were sintered using the same conditions.

\section{Materials characterization}

Low field permittivity of the composites was measured at a fixed frequency of $f=1 \mathrm{kHz}$ using an HP 4284A LCR meter. Low field direct $d_{33}$ constants were obtained using a Berlincourt type $d_{33}$ meter (PM3000, PiezoTest, UK). Bipolar polarization of the cured composite samples was measured using a modified Sawyer Tower circuit. The polarization was measured by applying a triangular voltage waveform using a Trek P0621P $30 \mathrm{kV}$ amplifier. The frequency of the applied voltage signal was $1 \mathrm{~Hz}$ for the DEP structured composites and $0.1 \mathrm{~Hz}$ for the 1-3 composites and PZT ceramics for polarization measurements and $0.1 \mathrm{~Hz}$ for all samples for the strain measurements.
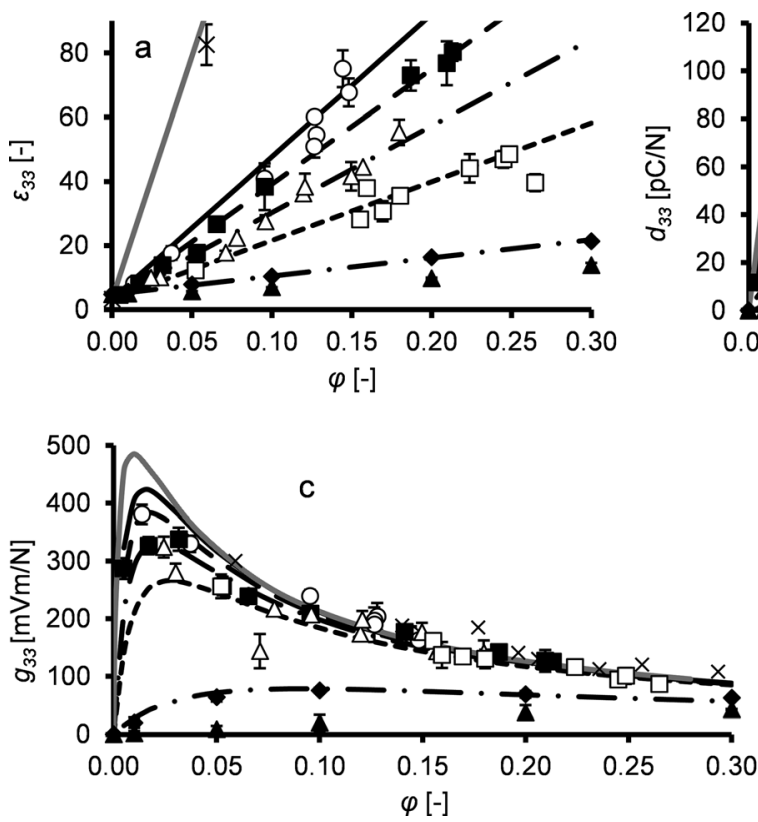

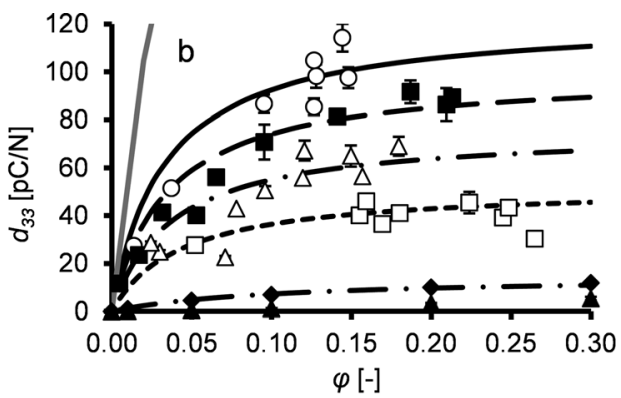

O R_eff $=108 \longrightarrow$ -

- R_eff $=85-$ - $\mathbf{\text { _eff }}=0.235$

$\triangle$ R_eff $=63 \quad$ - $\cdot$ Г_eff $=0.178$

$\square \quad$ R_eff $=46 \quad----\Gamma \_$eff $=0.122$

- $A R=1(D E P) \quad$ - 「_eff $=0.031$

- $A R=1(0-3) \quad$ r_eff $=1(1-3)$
FIG. 2. Relative permittivity (a), piezoelectric charge constants (b), and piezoelectric voltage constants (c) of 1-3 fiber composites and dielectrophoretically aligned composites as a function of particle volume fraction for different $R_{\text {eff }}$ compared to model values using Eqs. (6) and (7) for $\varepsilon$ and $d_{33}$, respectively. The values for particle composites $(A R=1)$ were taken from Ref. 8. 


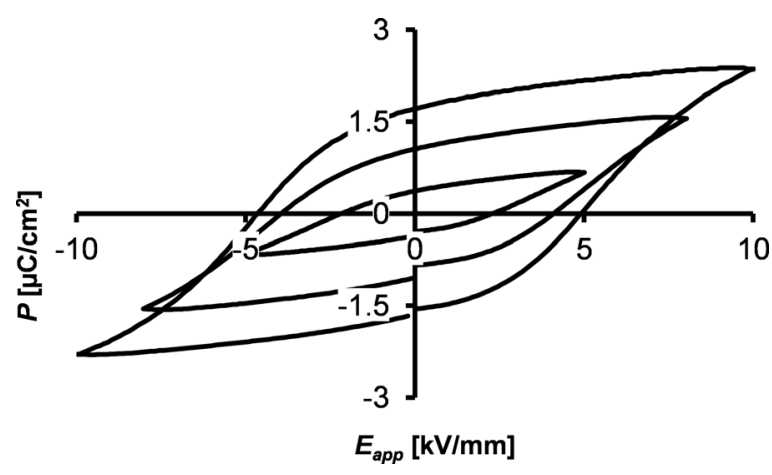

FIG. 3. Typical polarization hysteresis loop of a DEP aligned composites. Composite properties are $\varphi=0.2$ and $R_{\text {eff }}=85$.

Simultaneously, the strain hysteresis was measured using a custom made sample holder fitted with a linear variable differential transformer (LVDT) connected to a lock-in amplifier (Stanford Research SR830). The samples were subsequently poled at $8 \mathrm{kV} / \mathrm{mm}$ at $90{ }^{\circ} \mathrm{C}$ in a silicone oil bath for $30 \mathrm{~min}$. The poling field was only removed after cooling the samples to room temperature. After poling, unipolar polarization and strain measurements were done using the same setup as for the bipolar measurements. Low field dielectric measurements were performed at $1 \mathrm{kHz}$ using an impedance meter (HP 4284A). Impedance measurements were made using an HP4194a impedance analyzer and a compensated 16334A contact fixture. After piezoelectric characterization, optical micrographs of the composites cross sections were made. These were analyzed to assess the average orientation of the short fibers in the composites $(\theta)$. Per sample, 4 micrographs were taken at different depths of the composite and image analysis using the method described in Ref. 8 was used to obtain the average orientation angle of the fibers.

\section{RESULTS}

\section{Dielectric and piezoelectric properties at low fields}

The dielectric and piezoelectric properties as a function of PZT volume fraction of composites with different $R_{\text {eff }}$ are presented in Figure 2. The corresponding curves are modeled using Eqs. (6) and (7) for $\varepsilon$ and $d_{33}$, respectively, using the corresponding values for $\Gamma_{\text {eff }}$. The relation between $R_{\text {eff }}$ and $\Gamma_{\text {eff }}$ is described in detail in Ref. 11. The $\varepsilon$ and $d_{33}$ values for these DEP structured composites are higher than traditional 0-3 composites but do not reach the values for low volume fraction 1-3 composites with continuous fibers. ${ }^{11,15,19}$ The $g_{33}$ values for the DEP structured composites are, however, comparable to the 1-3 composite values.

\section{Polarization and strain at high electric fields}

The typical polarization behavior as a function of electric field of a DEP structured composite is presented in Figure 3. The microstructural properties of this composite were $\varphi=0.2, A R=11, \theta=68^{\circ}$, yielding an effective size ratio of $R_{\text {eff }}=85$. In Figure 4, the polarization of two DEP composites with $\varphi=0.2, A R=11, \theta=68^{\circ}, R_{\text {eff }}=85$ and $\varphi=0.2$, $A R=6, \theta=49^{\circ}, R_{\text {eff }}=45$, is compared to that of a comparable 1-3 composite with continuous fibers and bulk PZT5A4 ceramic. The polarization as a function of applied electric field of DEP structured composites is similar in form to the bulk PZT5 hysteresis but with much higher coercive fields compared to bulk PZT and 1-3 PZT-polymer composites. Typical coercive fields of around $4-6 \mathrm{kV} / \mathrm{mm}$ were found for the aligned short fiber composites at an applied field of $E_{\text {app }}=10 \mathrm{kV} / \mathrm{mm}$, depending on the aspect ratio and average orientation angles of the fibers in the composite.

The bipolar strain hysteresis as a function of applied field is presented in Figure 5. The shape of the strain loops of the DEP aligned composite materials is slightly asymmetrical. At
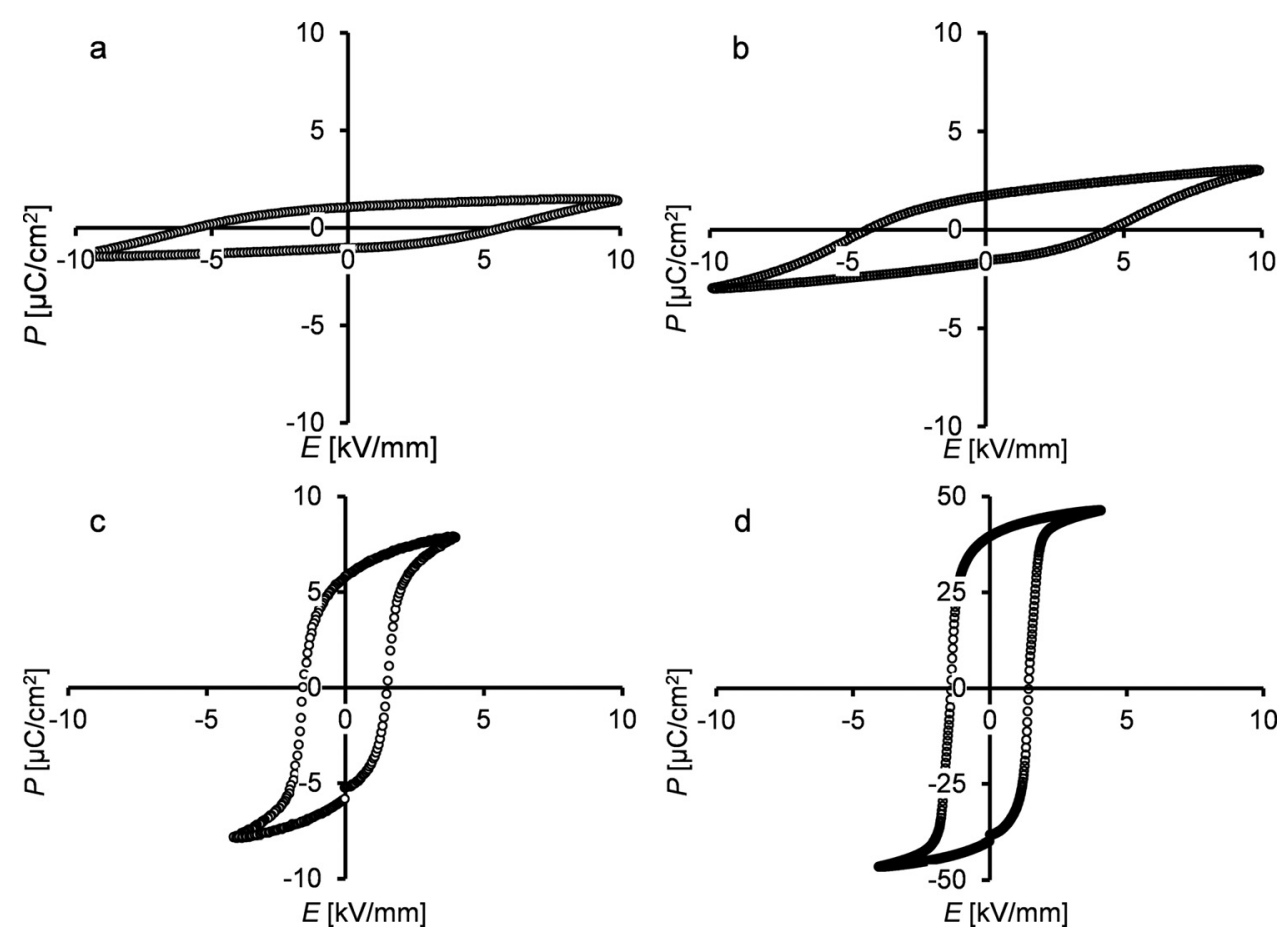

FIG. 4. Comparison between a DEP aligned fiber composites $(\varphi=0.2$, $\left.R_{\text {eff }}=45\right)$ and $\left(\varphi=0.2, R_{\text {eff }}=85\right), 1-3$ fiber composite $(\varphi=0.2)$, and PZT 5A4 bulk ceramic. 


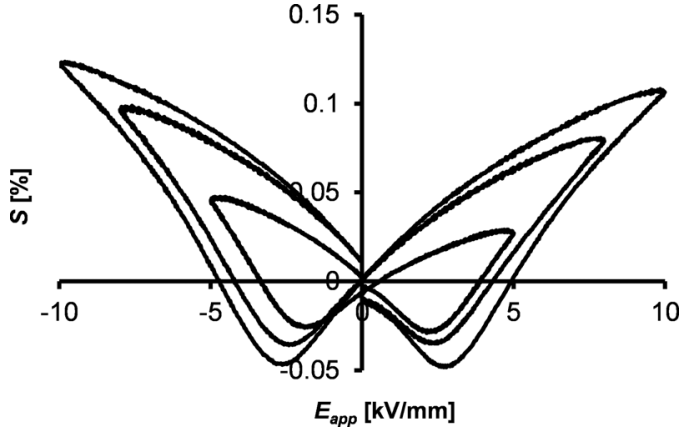

FIG. 5. Typical evolution of bipolar strain hysteresis loop of a DEP structured composite ( $\left.\varphi=0.2, R_{\text {eff }}=85\right)$.

low fields, this effect is most visible. The strain response is comparable to the response of 1-3 composite and bulk PZT materials (Figure 6). The coercive field for the DEP structured composites is higher while the maximum strain of the composite is lower than that of the 1-3 composite and that of the bulk material.

The unipolar polarization (Figure 7) and strain hysteresis (Figure 8 ) in the positive poling direction were measured for poled composites and the corresponding values for $\varepsilon_{33}$ and $d_{33}$ were calculated by $\varepsilon_{0} \varepsilon_{r}(E)=\frac{d P}{d E}$, and $d_{33}(E)=\frac{d S}{d E}$. Both $\varepsilon_{33}$ and $d_{33}$ increase with applied field up to a maximum, after which the values decrease for all systems. The position of this maximum is different for the different materials. The PZT ceramic reaches its maximum at around $E_{\text {app }}=1 \mathrm{kV} / \mathrm{mm}$ while for the composites, the maximum is reached at a higher applied field.

\section{Impedance measurements around resonance}

Impedance measurement results are presented in Figure 9. The half wave thickness resonance characteristics are shown for a PZT5A4 disk and 1-3 composite $(\varphi=0.24)$ and two different DEP structured composites. The DEP structured composites contain inclusions with different average aspect ratios, a "high AR" sample $\left(\varphi=0.15, A R_{\text {eff }}=11\right.$, $\left.R_{\text {eff }}=116\right)$ and "low AR" sample $\left(\varphi=0.24, A R_{\text {eff }}=6\right.$, $R_{\text {eff }}=41$ ). The range of the resonance frequencies that were measured lie between 1 and $7 \mathrm{MHz}$ and depend on sample geometry and material properties.

\section{DISCUSSION}

\section{Electric field distributions in the composites}

Using Eq. (4), the effects of aspect ratio and particle length/inter-particle matrix thickness ratio on the normalized electric field acting on an ellipsoidal PZT particle for a fixed relative permittivity values of $\varepsilon_{1}=3.2$ and $\varepsilon_{2}=1550$ are given in Figure 10. These permittivity values are low-field permittivity values of the PU matrix and PZT fibers, respectively. ${ }^{11}$

As follows from Eq. (5), the electric field values are greatest in the matrix region between the fibers. The analytical results for fixed relative permittivity values of $\varepsilon_{1}=3.2$ and $\varepsilon_{2}=1550$ are presented in Figure 11.

The results in Figures 10 and 11 show that the electric field acting on the particle increases when the aspect ratio of the particles increases and when the inter-particle distance decreases. However, for small aspect ratio particles, the concentration in electric field between 2 closely connected particles increases dramatically. The results from Eq. (5) indicate that electric fields between closely spaced filler particles can be orders of magnitude higher than the applied field. If the aspect ratio of the filler particles is high enough, however, the peak electric field between the particles drops. The results in Figure 11 are consistent with reports that breakdown electric fields are lower in the direction of alignment for DEP aligned composites with low $A R$ particles
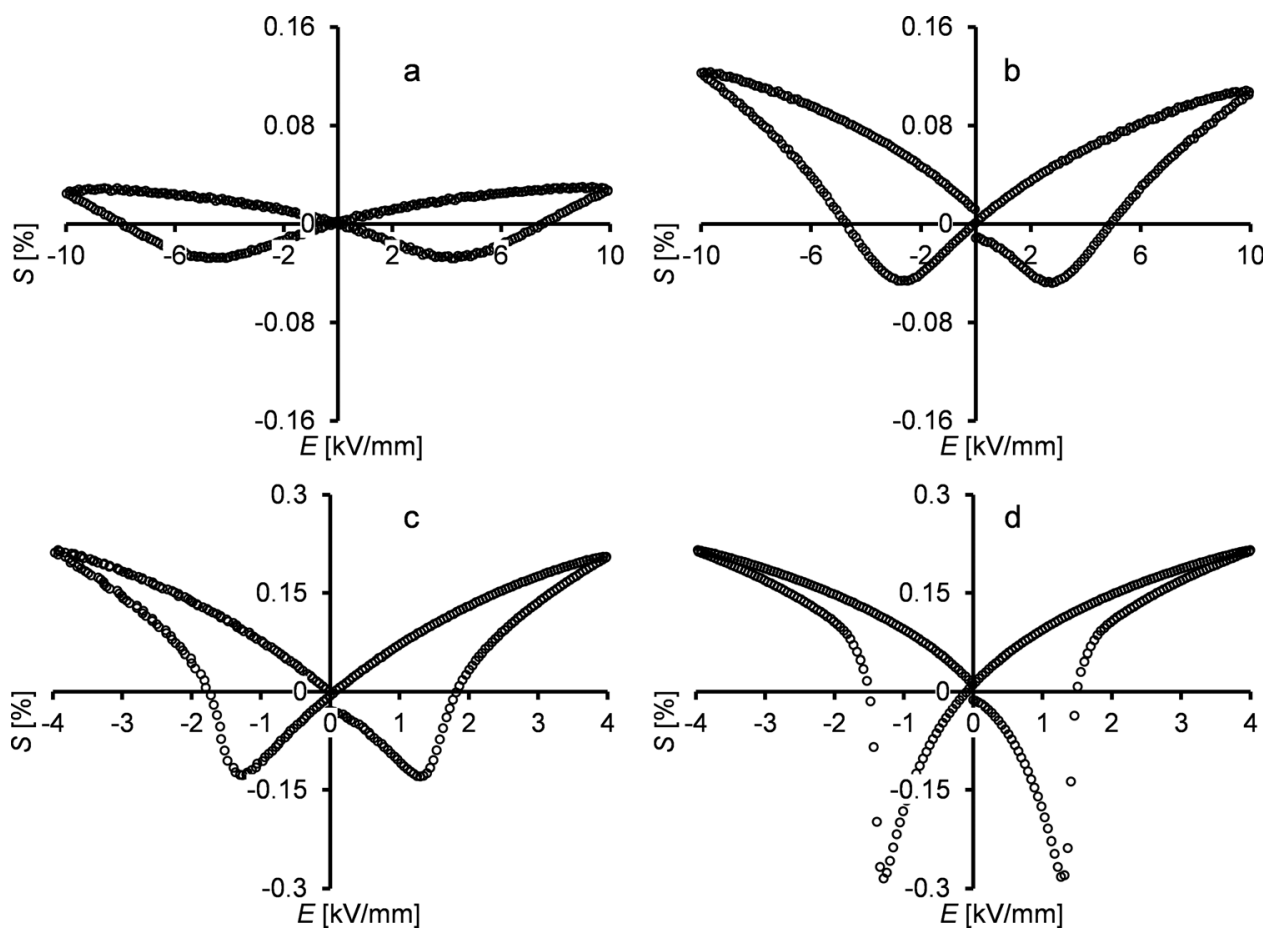

FIG. 6. Comparison between a DEP aligned fiber composites ((a) $\varphi=0.2$, $\left.R_{\text {eff }}=45\right)$ and ((b) $\left.\varphi=0.2, R_{\text {eff }}=85\right)$, 1-3 fiber composite ((c) $\varphi=0.2)$, and PZT 5A4 bulk ceramic (d). Note the scale difference in $\mathrm{x}-$, and $\mathrm{y}$-axes for (a) and (b) compared to (c) and (d). 

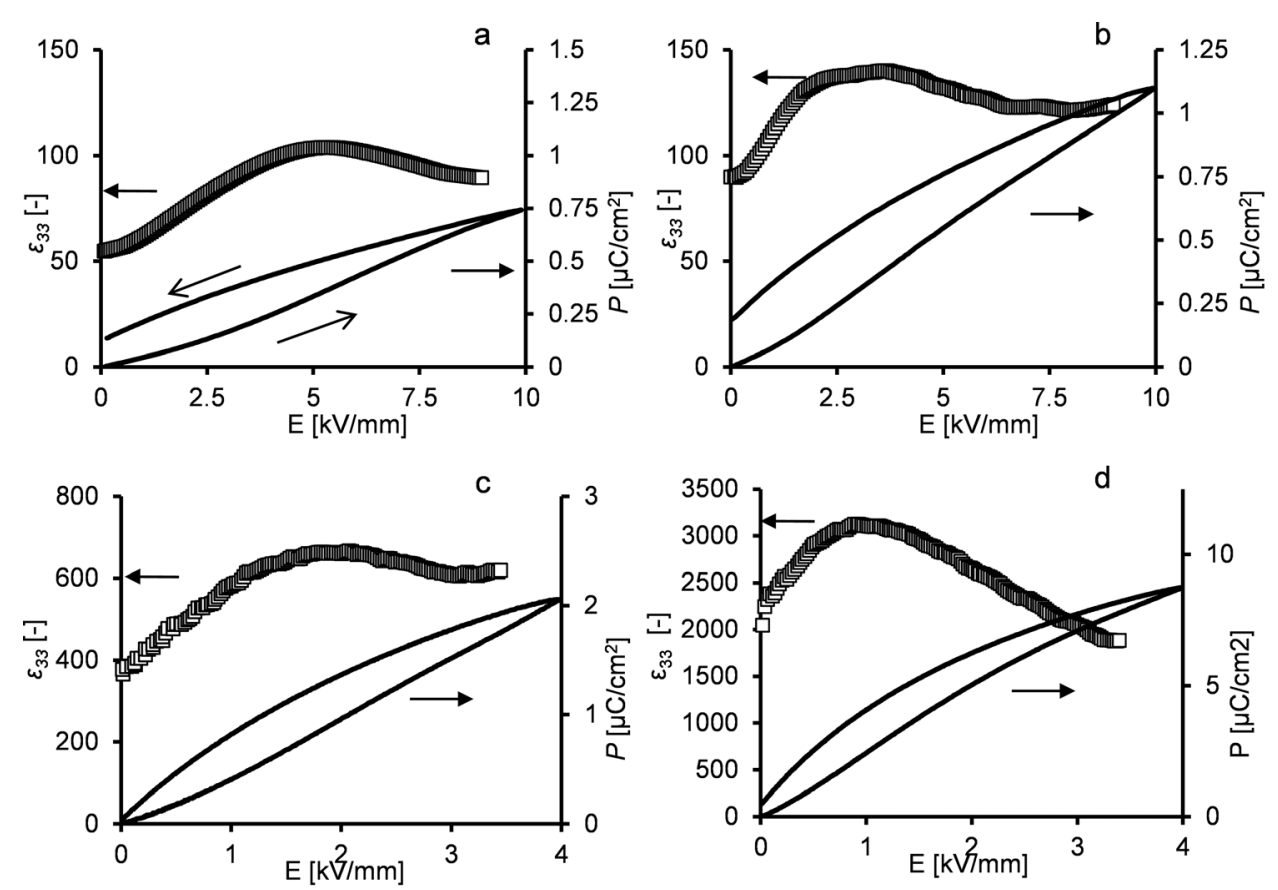

FIG. 7. Unipolar polarization hysteresis of DEP structured composites ((a) $\left.\varphi=0.2, \quad R_{\text {eff }}=45\right)$ and ((b) $\varphi=0.2$, $\left.R_{\text {eff }}=85\right), \quad 1-3$ fiber composite ((c) $\varphi=0.2$ ), and PZT 5A4 bulk ceramic (d) and associated values of the relative permittivity, $\varepsilon_{33}$. In (a), the open arrows indicate the direction of applied field. compared to composites with $\mathrm{x}-\mathrm{y}$ aligned or randomly dispersed particles ${ }^{20-22}$ and may be interesting for nanowire based composite capacitors. ${ }^{9}$

\section{High field properties of PZT-PU composites}

The polarization of the DEP structured quasi 1-3 composites is always lower than the polarization of the 1-3 composites and bulk ceramics at the same applied electric field. This lower polarization can be expected from the analysis in "Electrical field distributions in the composites" section. Using Eqs. (8) and (9), the polarization and strain of the DEP aligned chains of PZT particles can be extracted. These values can be regarded as an upper bound for the DEP structured composites with a corresponding $R_{\text {eff. }}$. The extracted values are presented in Figure 12. The maximum achieved strain of $0.15 \%$ for $R_{\text {eff }}=85$ is (Figure 8 ) is comparable to PZT ceramics operated at $E_{a p p}=2.5 \mathrm{kV} / \mathrm{mm}$. In practice, this electric field level is often taken as an operational limit of applied field to ensure adequate lifetime of the ceramics. The fatigue characteristics of the DEP structured composites operated at $E_{a p p}=10 \mathrm{kV} / \mathrm{mm}$ have not been tested, but if a polymer with sufficiently high electrical strength is used as a matrix, the practical operation strain limit can be made comparable to that of PZT. However, the importance of the $R_{\text {eff }}$ value is illustrated in Figure 12 when comparing the composite with $R_{\text {eff }}=85$ to the composite with $R_{\text {eff }}=45$, which does not achieve one third of its maximum strain. The electric field ratio acting on the particles determines the achievable strain for a fixed applied field. The electric field ratio is
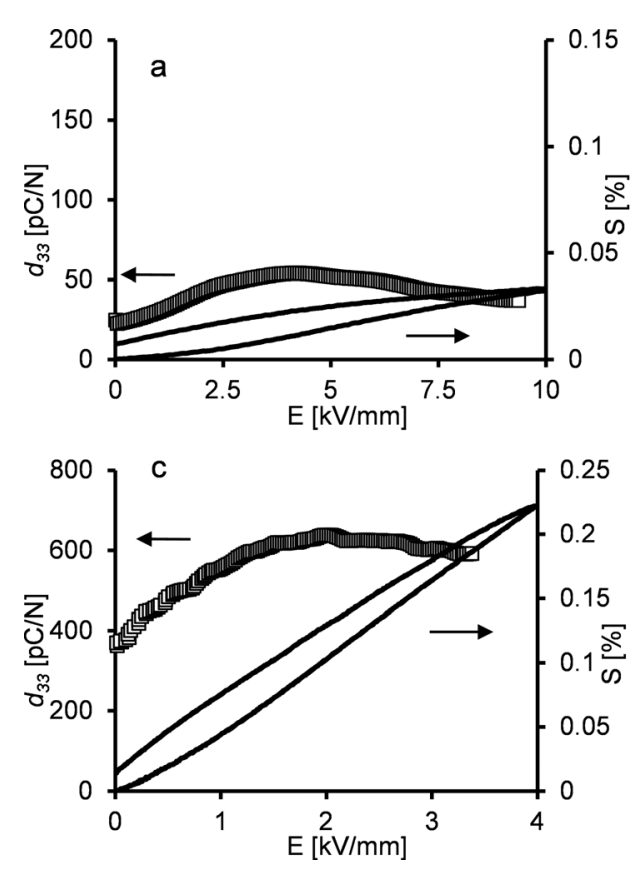
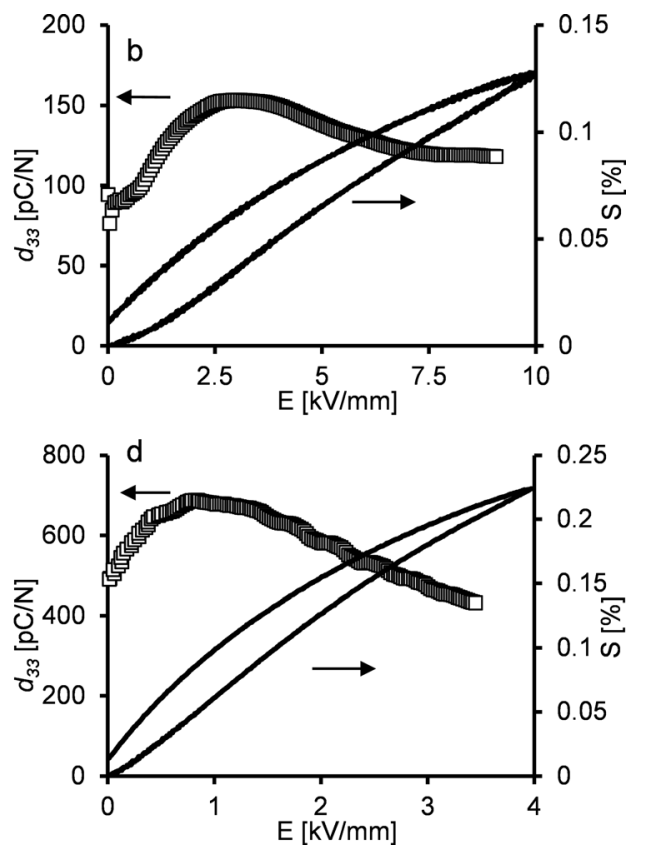

FIG. 8. Unipolar strain hysteresis of DEP structured composites ((a) $\varphi=0.2$, $\left.R_{\text {eff }}=45\right)$ and ((b) $\left.\varphi=0.2, R_{\text {eff }}=85\right)$, 1-3 fiber composite ((c) $\varphi=0.2)$ and PZT 5A4 bulk ceramic (d) and associated $d_{33}$ values. 


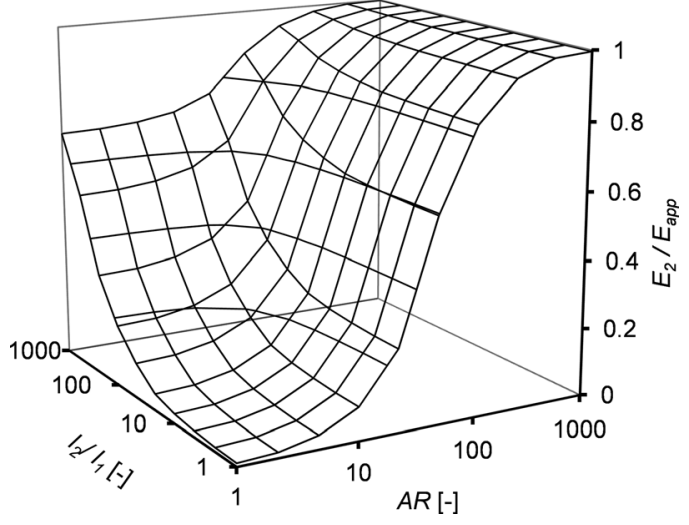

FIG. 9. Impedance magnitude and phase around resonance of DEP structured composites ((a) $\varphi=0.24, R_{\text {eff }}=41$ ) and ((b) $\left.\varphi=0.15, R_{\text {eff }}=116\right), 1-3$ fiber composite ((c) $\varphi=0.24)$ and PZT 5A4 bulk ceramic disk (d). The KLM fits for each sample are represented by the solid (magnitude) and dashed (phase) lines.

retained at high fields resulting in an increase in $\varepsilon_{33}$ and $d_{33}$ of the composites. Both $\varepsilon_{33}$ and $d_{33}$ exhibit a maximum in the DEP aligned composites. This result indicates that the value of $\Gamma_{\text {eff }}$ may change at high fields. The position of the maximum is different for composite with a different value of $R_{\text {eff, }}$, shifting to a lower $E_{a p p}$ for higher $R_{\text {eff. }}$ No signs of breakdown were observed in the composites at $E_{a p p}=10 \mathrm{kV} /$ $\mathrm{mm}$ over the whole range of $R_{\text {eff }}$ that was tested. This effect may be because the composites with a higher $R_{\text {eff }}$ all tended to have higher $A R$ particles. A higher $A R$ decreases $E_{1}$, avoiding breakdown (see Eq. (5) and Figure 11).

The difference in maximum polarization between bulk PZT5A4 ceramics (see Figure 7) and the fiber properties (Figure 12) derived from composite materials is in part related to the clamping effect caused by the matrix in the low volume fraction composites. ${ }^{15,23}$ Therefore, it must be

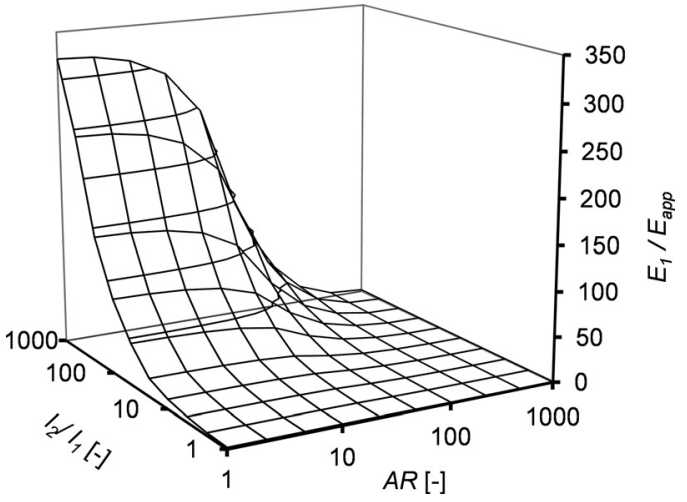

FIG. 10. Electric field ratio acting on an ellipsoidal PZT particle as a function of aspect ratio, $A R$ and different particle spacing, $R$ using Eq. (4).

noted that the fiber properties presented here are the effective fiber properties in the composite at a volume fraction of $\varphi=0.2$ and single fiber properties will differ. ${ }^{23}$ This volume fraction is comparable to the volume fractions of the DEP structured composites. The measured coercive field of unconstrained PZT fibers will also be slightly higher than the coercive field of the low $\varphi$ 1-3 composite due to clamping. ${ }^{24}$ The DEP structured composites show higher coercive and saturation electric fields than the 1-3 composites. In general, $\frac{d P}{d E}$ is smaller for the DEP aligned composites and is a result of $\Gamma_{\text {eff }}<1$ (see Eq. (4)). The coercive field of the composites is also related to $\Gamma_{\text {eff }}$ and results in $E_{c D E P}>E_{c 1-3}$. The maximum strain (Figure 6) in the positive poling direction of the 1-3 composites is higher than that of the bulk ceramic and relatively high compared to values reported in literature. ${ }^{15,25}$ This is attributed to the uniaxial strain state in the fibers, which have relatively high aspect ratio and small lateral dimensions, especially for the continuous 1-3 fiber composites. This effect was also previously reported for small
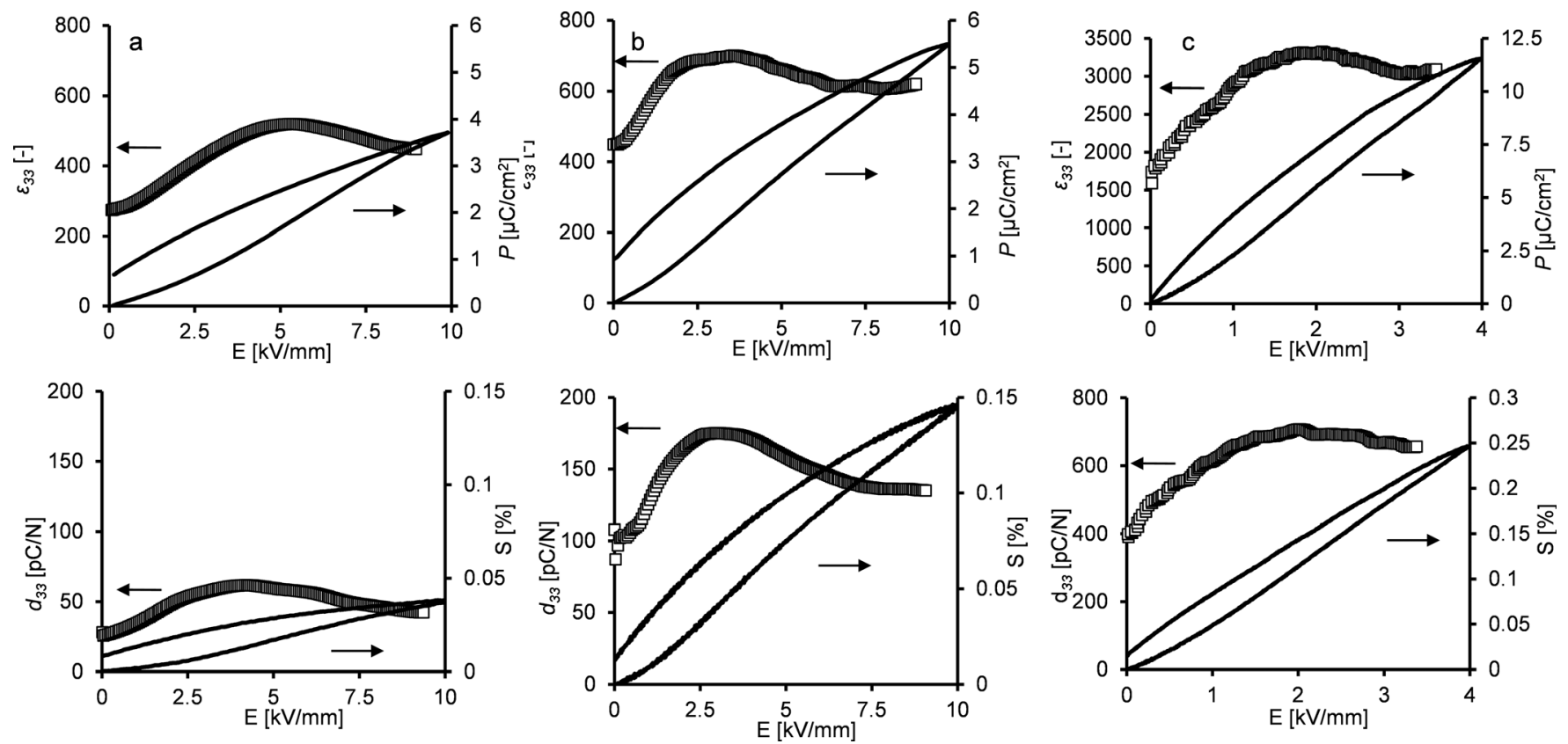

FIG. 11. The effective electric field concentration in the matrix layer between two adjacent particles as a function of particle dimensions and inter-particle distance. 


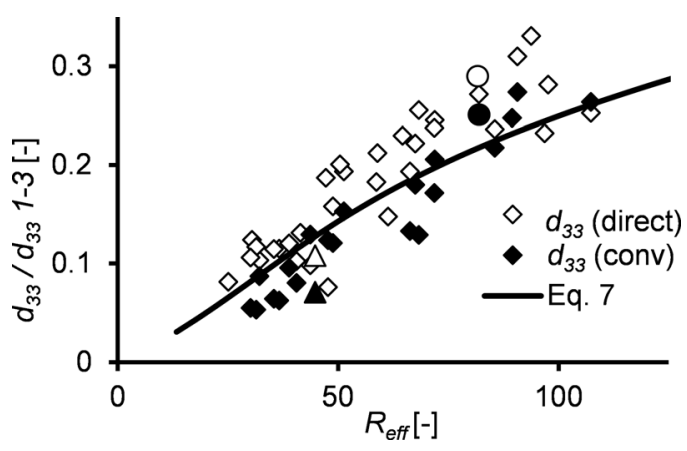

FIG. 12. Unipolar polarization and strain hysteresis of DEP structured composites ((a) $\left.R_{\text {eff }}=45\right)$ and ((b) $R_{\text {eff }}=85$ ) and 1-3 fiber composite (c) and associated $\varepsilon_{33}$ and $d_{33}$ values. The polarization and strain values were normalized for the PZT phase in the composites using Eqs. (8) and (9). The bulk PZT values can be found in Figures 8(d) for comparison.

diameter fibers ${ }^{15}$ because the stress transfer from fiber to matrix depends on the fiber surface area. ${ }^{26}$ Alternatively, a relatively low maximum compressive strain at the point of polarization reversal is seen for 1-3 composites and DEP structured composites. This is again caused by clamping of the high aspect ratio fibers, ${ }^{15}$ since depolarization of PZT is known to be aided by compressive stress ${ }^{27}$ leading to lower compressive strains at polarization reversal. ${ }^{23,24}$ In the DEP structured composites, an even smoother strain reversal behavior is seen due to the distribution of fiber aspect ratios and orientations. When the polarization of each particle in the composite is oriented in the same direction, the contributions are averaged. But when the polarization is switched this difference becomes more visible. In the region close to the coercive field of the composite, the polarization of some of the particle inclusions will be switched while others are still polarized in the poling direction. The DEP structured composites all exhibited asymmetric strain polarization behavior as the example in Figure 5. No clear virgin polarisation curve could be measured for any of the DEP aligned composites, suggesting (partial) poling during manufacturing.

In the cured DEP aligned composites, the electric field in the matrix region between two adjacent particles is dramatically increased because the particles are brought in close proximity of each other by the DEP force. The permittivity increases as a function of the field component in the matrix phase, $E_{1}^{\prime}$. This component is a function of the particle dimension and inter-particle distance as can be seen in Figure 11. The electric field intensities for sample composites can be estimated based on the low field values using Eq. (5). They are on the order of $50<E_{1}^{\prime} / E_{a p p}<115$ if the permittivity of the PU phase is kept constant at $\varepsilon_{1}=3.2$ and no loss is considered (see Figure 11). This would yield electric fields of up to $E_{1}^{\prime}=1000 \mathrm{kV} / \mathrm{mm}$ at $E_{a p p}=10 \mathrm{kV} / \mathrm{mm}$. This is about 50 times the typical breakdown field of bulk polyurethane. However, no breakdown is observed in the composites during these measurements. The breakdown field of nano-scale interfaces is known to be much higher than breakdown field of bulk material, ${ }^{20}$ which might partly explain why the composite samples do not break down at these applied voltages. If the composite features can be scaled down, f.i. using nanowires as particle inclusions ${ }^{28,29}$ the fea- ture size will decrease. This will be beneficial in two ways. The total applied voltage will decrease and the inter particle distance will decrease for constant $R_{\text {eff. }}$ This effect will result in an even thinner inter-particle matrix layer with a higher dielectric breakdown electric field. ${ }^{20}$

In Figure 13, the converse $d_{33}$ of the composites is compared to the direct piezoelectric measurements, which were measured on a Berlincourt $d_{33}$ meter (i.e., the values in Figure 2(b)). The DEP structured composite $d_{33}$ values relative to 1-3 composite $d_{33}$ values of the same volume fraction are plotted, along with the theoretical values calculated using Eq. (7). The values are comparable, though the converse measurement yields slightly lower values than the direct measurement. The maximum converse $d_{33}$ is compared to the low-field converse effect in Figure 14. The high field $d_{33}$ data are compared to the calculated $d_{33}$ of 1-3 composites at the corresponding electric field using $E_{2}=\Gamma_{e f f} E_{a p p}$, where the low field value for $\Gamma_{\text {eff }}$ was used as an approximation for the applied field ratio on the particle inclusions. The relative $d_{33}$ at high fields is slightly higher than the low field ratio for composites, suggesting a modest increase of $\Gamma_{\text {eff }}$ at higher applied fields.

In general, DEP structured PZT-polymer composites with high aspect ratio particle inclusions have low field piezoelectric constants which are much higher than those of traditional 0-3 granular particle composites and have piezoelectric voltage constants which are comparable to 1-3 composites (see Figure 2). This high $g_{33}$ is beneficial when the material is used as a transducer receiver. At high applied electric fields, the $d_{33}$ increases towards a maximum at around $2.5-5 \mathrm{kV} / \mathrm{mm}$ and subsequently decreases slightly. Because of its higher maximum applied field, the electromechanical strain that can be achieved is closer to the ceramic and 1-3 composite values than the $d_{33}$ values alone would suggest.

\section{Thickness coupling factors of DEP structured composites}

Using the data of the impedance plots for frequencies around the resonance frequency for three composites and one reference bulk PZT sample (Figure 9), the thickness coupling factors were calculated using Eq. (10). It is known that for highly damped systems the use of Eq. (10) may lead to inaccuracies in the calculated $k_{t}$ values. ${ }^{30}$ Therefore, a

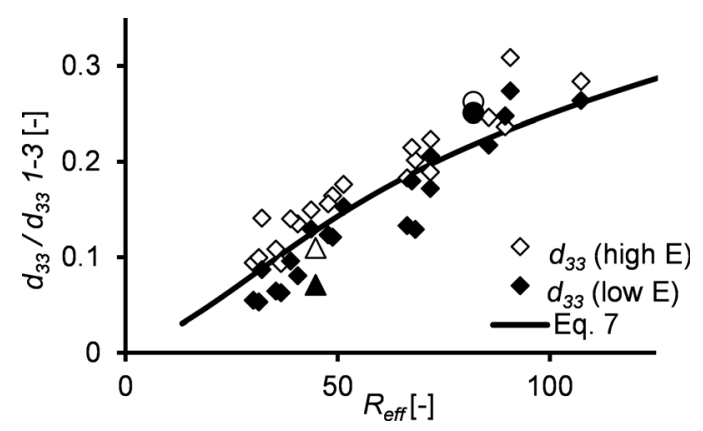

FIG. 13. Converse $d_{33}$ at field low conditions $\left(E_{a p p}=0.1 \mathrm{kV} / \mathrm{mm}\right)$, compared to the direct $d_{33}$. The triangles are values for the example composite with $R_{\text {eff }}=45$ and circles are values for the composite with $R_{\text {eff }}=85$. 

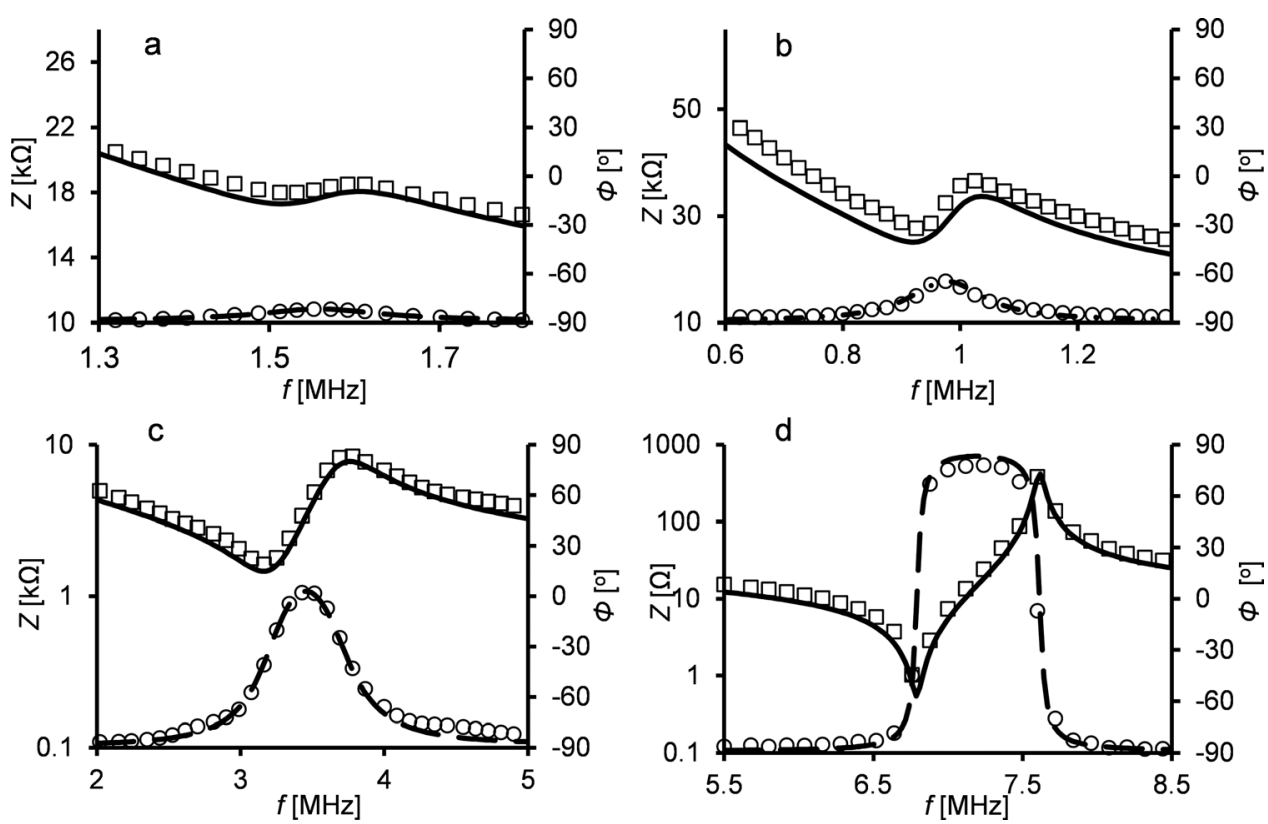

FIG. 14. Maximum $d_{33}$ at high field conditions compared to the $d_{33}$ under low field conditions (converse effect). The triangles are values for the example composite with $R_{\text {eff }}=45$ and circles are values for the composite with $R_{\text {eff }}=85$. comparison to the calculated coupling factors was made by fitting a custom implementation of the KLM (Krimholtz, Leedom, Matthaei) equivalent circuit model ${ }^{31,32}$ to the impedance data for $k_{t}$. The mechanical quality factor, $Q_{m}$, was separately estimated using the technique outlined in Ref. 33. The fitted impedance curves are presented Figure 9. In Table I, calculated $k_{t}$ values using Eq. (10) and the fitted $k_{t}$ values are compared. The values calculated using Eq. (10) slightly underestimate $k_{t}$ compared to the fitted values from the equivalent circuit models.

The "low AR" DEP structured composites show a thickness coupling factor which is comparable to that of PVDF polymer and "high AR" DEP materials are comparable to PVDF-TrFE polymers. The extremely low $Q_{m}$ of the DEP structured composites are possibly the result of the size distribution of the inclusions, causing local variations in stiffness and piezoelectric coefficients within the material. These local differences generally broaden the resonance peak of the sample. ${ }^{34}$ This effect has been reported for unstructured piezoelectric PZT-polymer composites with high PZT volume fractions. ${ }^{35}$ In the 1-3 composite, the same effects are caused by local variations in fiber packing density due to the processing technique used (see experimental section). Thus, resonance measurements show that the DEP aligned composites posses a fair amount of electromechanical coupling for such low volume fractions of PZT and bandwidth is high due to mechanical damping. The high coupling

TABLE I. Calculated and fitted thickness coupling factor $\left(k_{t}\right)$ and mechanical quality factor $\left(Q_{m}\right)$ and calculated thickness frequency constant $\left(N_{3}^{D}\right)$ for the DEP aligned composites, 1-3 composite, and PZT5A disk.

\begin{tabular}{lccccc}
\hline \hline Material & $\begin{array}{c}k_{t} \\
\text { (Eq. (10)) }\end{array}$ & $\begin{array}{c}k_{t} \mathrm{KLM} \\
\mathrm{fit}^{31}\end{array}$ & $Q_{m}{ }^{33}$ & $\begin{array}{c}Q_{m} \mathrm{KLM} \\
\mathrm{fit}^{31}\end{array}$ & $\begin{array}{c}N_{3}^{D} \\
{[\mathrm{~m} / \mathrm{s}]}\end{array}$ \\
\hline Low AR, DEP composite & 0.10 & 0.13 & 6.7 & 8.9 & 1100 \\
High AR DEP composite & 0.26 & 0.28 & 5.9 & 6.5 & 1220 \\
1-3 Composite & 0.47 & 0.52 & 6.5 & 8.2 & 1390 \\
PZT5A4 disk & 0.49 & 0.49 & 123 & 133 & 2030 \\
\hline \hline
\end{tabular}

factors are consistent with the high $d_{33}$ values that were measured for the composites at low frequency. Also, electrical loss remains relatively low $\left(\tan \delta_{\mathrm{e}} \approx 0.02-0.04\right.$ in $\mathrm{MHz}$ frequency range). These electrical losses are much lower than for PVDF $\left(\tan \delta_{\mathrm{e}} \approx 0.24\right.$ in the $\mathrm{MHz}$ range). ${ }^{36}$ Also the lower frequency constant (see Table I) allows for thinner transducers compared to PZT devices. Therefore, the DEP composites could potentially be an interesting alternative for transducers which are now using piezoelectric polymers. These properties could make the composites interesting candidates for large area transducers. They are especially interesting if integration with existing complex shaped structures is needed and a large shaping freedom is required., ${ }^{2,12}$ Analogous to continuous PZT fiber composites, ${ }^{37}$ DEP structured composites can also be employed in-plane by making use of interdigitated electrodes. ${ }^{38}$ This electrode structure creates the possibility to manufacture thin and compliant piezoelectric transducer coatings with higher coupling factors at lower PZT volume fractions than conventional 0-3 composite coatings based on randomly dispersed particles such as mentioned in Ref. 2.

\section{CONCLUSIONS}

DEP structured PZT-polymer composites with high aspect ratio particle inclusions have low field piezoelectric constants which are much higher than those of traditional 03 granular particle composites, especially at low volume fractions. They can achieve piezoelectric voltage constants which are comparable to 1-3 composites with continuous fibers. This high $g_{33}$ is beneficial when the material is used as a transducer receiver. Also significant electromechanical coupling at high frequencies was measured at resonance for DEP structured composites. Polarization, strain, and coercive field of the fibers were all found to be dependent on $R_{\text {eff }}$, the effective length of the fiber over the distance between the fibers. The electric field corresponding to a maximum of $\varepsilon_{33}$ and $d_{33}$ is dependent on $R_{\text {eff }}$, shifting to a lower $E_{a p p}$ for

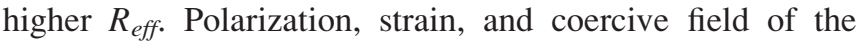


fibers are found to depend on $R_{\text {eff. }}$. For composites with well aligned high aspect ratio particles $\left(R_{\text {eff }} \sim 85\right)$, the electromechanical strain that can be achieved approaches the values for the ceramic and 1-3 fibrous composites. The observed dependences can be explained by a model taking into account the spatial arrangement of the particles and the material properties. If the thickness can be scaled down while retaining the high aspect ratio of the inclusions, for instance, by using PZT nanowires as the high aspect ratio particles, this type of composite can possibly be employed for transducers which are cost effective and relatively simple to process. These materials are especially interesting if integration with existing structures is needed, because the composites can be processed at low temperatures.

\section{ACKNOWLEDGMENTS}

This work was financially supported by the Smartmix funding program (Grant No. SMVA0607), as part of the program "Smart systems based on integrated Piezo". D. A. van den Ende acknowledges the help from the staff and students at the Centre for Dielectric Studies (CDS) at The Pennsylvania State University where part of the research was conducted.

${ }^{1}$ R. E. Newnham, D. P. Skinner, and L. E. Cross, Mater. Res. Bull. 13, 525 (1978).

${ }^{2}$ S. Egusa and N. Iwasawa, J. Mater. Sci. 28, 1667-1672 (1993).

${ }^{3}$ C. J. Dias and D. K. Das Gupta, IEEE Trans. Electr. Insul. 3, 706 (1996).

${ }^{4}$ R. E. Newnham, A. Safari, G. Sa-Gong, and J. Giniewicz, in IEEE International Ultrasonics Symposium (1984), p. 501.

${ }^{5} \mathrm{H}$. A. Pohl, Dielectrophoresis: The Behavior of Neutral Matter in Nonuniform Electric Fields (Cambridge University Press, Cambridge, 1978).

${ }^{6}$ C. A. Randall, S. Miyazaki, K. L. More, A. S. Bhalla, and R. E. Newnham, Mater. Lett. 15, 26-30 (1992).

${ }^{7}$ C. P. Bowen, R. E. Newnham, and C. A. Randall, J. Mater. Res. 13, 205 (1998).

${ }^{8}$ D. A. van den Ende, B. F. Bory, W. A. Groen, and S. van der Zwaag, J. Appl. Phys. 107, 024107 (2010).

${ }^{9}$ H. Tang, Y. Lin, and H. A. Sodano, Adv. Energy Mater. 2, 469 (2012).

${ }^{10}$ H. Tang, Y. Lin, C. Andrews, and H. A. Sodano, Nanotechnology 22, 015702 (2011).

${ }^{11}$ D. A. van den Ende, S. E. van Kempen, X. Wu, W. A. Groen, C. A. Randall, and S. van der Zwaag, J. Appl. Phys. 111, 124107 (2012).
${ }^{12}$ J. Feenstra and H. A. Sodano, J. Appl. Phys. 103, 124108 (2008).

${ }^{13}$ C. J. F. Böttcher, O. C. van Belle, P. Bordewijk, and A. Rip, Theory of Electric Polarization (Elsevier Scientific, 1978).

${ }^{14}$ A. Sihvola, Electromagnetic Mixing Rules and Applications (The Institution of Electrical Engineers, London, UK, 1999).

${ }^{15}$ L. J. Nelson, C. R. Bowen, R. Stevens, M. Cain, and M. Stewart, Proc. SPIE 5053, 544 (2003).

${ }^{16}$ H. L. W. Chan and J. Unsworth, IEEE Trans. Ultrason. Ferroelectr. Freq. Control 36, 434 (1989).

${ }^{17}$ M. Kim, J. Kim, and W. Cao, Appl. Phys. Lett. 87, 132901 (2005).

${ }^{18}$ A. Meitzler, D. Berlincourt, G. Coquin, F. Welsh, H. Tiersten, and A. Warner, IEEE Standards on Piezoelectricity, ANSI/IEEE Std 176-1978 (The Institute of Electrical and Electronics Engineers, 1987).

${ }^{19}$ H. P. Savakus, K. A. Klicker, and R. E. Newnham, Mater. Res. Bull. 16, 677 (1981).

${ }^{20}$ V. Tomer and C. A. Randall, J. Appl. Phys. 104, 074106 (2008).

${ }^{21}$ V. Tomer, C. A. Randall, G. Polizos, J. Kostelnick, and E. Manias, J. Appl. Phys. 103, 034115 (2008).

${ }^{22}$ J. E. Martin, C. P. Tigges, R. A. Anderson, and J. Odinek, Phys. Rev. B 60, 7127 (1999).

${ }^{23}$ A. Belloli, J. Heiber, F. Clemens, and P. Ermanni, J. Intell. Mater. Syst. Struct. 20, 355 (2009).

${ }^{24}$ D. Zhou, M. Kamlah, and D. Munz, J. Eur. Ceram. Soc. 25, 425-432 (2005).

${ }^{25}$ C. R. Bowen, R. Stevens, L. J. Nelson, A C. Dent, G. Dolman, B. Su, T. W. Button, M. G. Cain, and M. Stewart, Smart Mater. Struct. 15, 295 (2006).

${ }^{26}$ Q. M. Zhang, W. Cao, H. Wang, and L. E. Cross, J. Appl. Phys. 73, 1403 (1993).

${ }^{27}$ T. Fett and D. Muntz, Adv. Eng. Mater. 5, 718 (2003).

${ }^{28}$ H. Tang, Y. Lin, and H. A. Sodano, Proc. SPIE 7978, 79780 S (2011).

${ }^{29}$ C. Andrews, Y. Lin, H. Tang, and H. A. Sodano, J. Int. Mater. Syst. Struct. 22, 1879 (2011)

${ }^{30}$ F. S. Foster, L. K. Ryan, and D. H. Turnbull, IEEE Trans. Ultrason. Ferroelectr. Freq. Control 38, 446 (1991).

${ }^{31}$ R. Krimholtz, D. A. Leedom, and G. L. Matthaei, Electron. Lett. 6, 398399 (1970).

${ }^{32}$ E. J. W. Merks, J. M. G. Borsboom, M. M. Voormolen, N. Bom, A. F. W. van der Steen, and N. de Jong, Ultrasonics 44, e705-e710 (2006).

${ }^{33}$ L. N. Bui, H. J. Shaw, and L. T. Zitelli, IEEE Trans. Son. Ultrason. 24, 331-336 (1977).

${ }^{34}$ M. P. Wenger, P. Blanas, C. J. Dias, R. J. Shuford, and D. K. Dasgupta, Ferroelectrics 187, 75-86 (1996).

${ }^{35}$ C. Dias, D. K. Das-Gupta, Y. Hinton, and R. J. Shuford, Sens. Actuators, A 37-38, 343-347 (1993).

${ }^{36}$ F. S. Foster, K. A. Harasiewicz, and M. D. Sherar, IEEE Trans. Ultrason. Ferroelectr. Freq. Control 47, 1363 (2000).

${ }^{37}$ A. A. Bent and N. W. Hagood, J. Int. Mater. Syst. Struct. 8, 903 (1997).

${ }^{38}$ D. A. van den Ende, H. J. van de Wiel, W. A. Groen, and S. van der Zwaag, Smart Mater. Struct. 21, 015011 (2012). 